\title{
Geometric Multispectral Camera Calibration
}

\author{
Johannes Brauers and Til Aach \\ Institute of Imaging \& Computer Vision, RWTH Aachen University, \\ Templergraben 55, D-52056 Aachen, Germany \\ Johannes.Brauers@lfb.rwth-aachen. de \\ http://www.lfb.rwth-aachen.de
}

\begin{abstract}
A large number of multispectral cameras uses optical bandpass filters to divide the electromagnetic spectrum into passbands. If the filters are placed between the sensor and the lens, the different thicknesses, refraction indices and tilt angles of the filters cause image distortions, which are different for each spectral passband. On the other hand, the lens also causes distortions which are critical in machine vision tasks. In this paper, we propose a method to calibrate the multispectral camera geometrically to remove all kinds of geometric distortions. To this end, the combination of the camera with each of the bandpass filters is considered as single camera system. The systems are then calibrated by estimation of the intrinsic and extrinsic camera parameters and geometrically merged via a homography. The experimental results show that our algorithm can be used to compensate for the geometric distortions of the lens and the optical bandpass filters simultaneously.
\end{abstract}

\section{Introduction}

Multispectral imaging considerably improves the color accuracy in contrast to conventional three-channel RGB imaging [1]: This is because RGB color filters exhibit a systematic color error due to production conditions and thus violate the Luther rule [2]. The latter states that, for a human-like color acquisition, the color filters have to be a linear combination of the human observer's ones. Additionally, multispectral cameras are able to differentiate metameric colors, i.e., colors with different spectra but whose color impressions are the same for a human viewer or an RGB camera. Furthermore, different illuminations can be simulated with the acquired spectral data after acquisition. A well-established multispectral camera type, viz., the one with a filter wheel, has been patented by Hill and Vorhagen [3] and is used by several research groups 4567 .

One disadvantage of the multispectral filter wheel camera are the different optical properties of the bandpass filters. Since the filters are positioned in the optical path, their different thicknesses, refraction indices and tilt angles cause a different path of rays for each passband when the filter wheel index position is changed. This causes both longitudinal and transversal aberrations in the acquired images: Longitudinal aberrations produce a blurring or defocusing effect 
in the image as shown in our paper in [8]. In the present paper, we consider the transversal aberrations, causing a geometric distortion. A combination of the uncorrected passband images leads to color fringes (see Fig. 3a). We presented a detailed physical model and compensation algorithm in 9]. Other researchers reported heuristic algorithms to correct the distortions 101112 caused by the bandpass filters. A common method is the geometric warping of all passband images to a selected reference passband, which eliminates the color fringes in the final reconstructed image.

However, the reference passband image also exhibits distortions caused by the lens. To overcome this limitation, we have developed an algorithm to compensate both types of aberrations, namely the ones caused by the different optical properties of the bandpass filters and the aberrations caused by the lens. Our basic idea is shown in Fig. 1] We interpret the combination of the camera with each optical bandpass filter as a separate camera system. We then use camera calibration techniques 13 in combination with a checkerboard test chart to estimate calibration parameters for the different optical systems. Afterwards, we warp the images geometrically according to a homography.

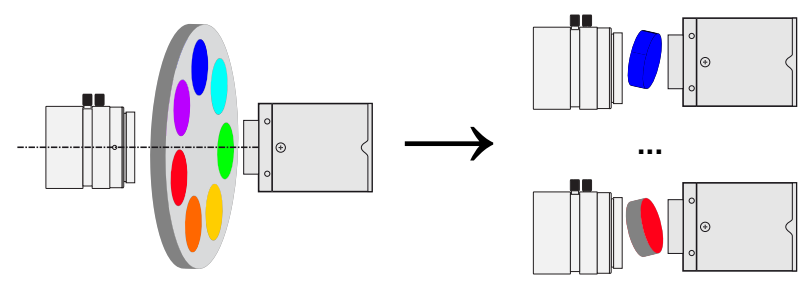

Fig. 1. With respect to camera calibration, our multispectral camera system can be interpreted as multiple camera systems with different optical bandpass filters

We have been inspired by two publications from Gao et. al [14,15, who used a plane-parallel plate in front of a camera to acquire stereo images. To a certain degree, our bandpass filters are optically equivalent to a plane-parallel plate. In our case, we are not able to estimate depth information because the base width of our system is close to zero. Additionally, our system exhibits seven different optical filters, whereas Gao uses only one plate. Furthermore, our optical filters are placed between optics and sensor, whereas Gao used the plate in front of the camera.

In the following section we describe our algorithm, which is subdivided into three parts: First, we compute the intrinsic and extrinsic camera parameters for all multispectral passbands. Next, we compute a homography between points in the image to be corrected and a reference image. In the last step, we finally compensate the image distortions. In the third section we present detailed practical results and finish with the conclusions in the fourth section. 


\section{Algorithm}

\subsection{Camera Calibration}

A pinhole geometry camera model 13 serves as the basis for our computations. We use

$$
\mathbf{x}_{n}=\frac{1}{Z}\left(\begin{array}{l}
X \\
Y
\end{array}\right)
$$

to transform the world coordinates $\mathbf{X}=(X, Y, Z)^{T}$ to normalized image coordinates $\mathbf{x}_{n}=\left(x_{n}, y_{n}\right)^{T}$. Together with the radius

$$
r_{n}^{2}=x_{n}^{2}+y_{n}^{2}
$$

we derive the distorted image coordinates $\mathbf{x}_{d}=\left(x_{d}, y_{d}\right)^{T}$ with

$$
\begin{aligned}
\mathbf{x}_{d} & =\left(1+k_{1} r_{n}^{2}+k_{2} r_{n}^{4}\right) \mathbf{x}_{n}+\left(\begin{array}{c}
2 k_{3} x_{n} y_{n}+k_{4}\left(r_{n}^{2}+2 x_{n}^{2}\right) \\
k_{3}\left(r_{n}^{2}+2 y_{n}^{2}\right)+2 k_{4} x_{n} y_{n}
\end{array}\right) \\
& =f\left(\mathbf{x}_{n}, \mathbf{k}\right) .
\end{aligned}
$$

The coefficients $k_{1}, k_{2}$ account for radial distortions and the coefficients $k_{3}, k_{4}$ for tangential ones. The function $f()$ describes the distortions and takes a normalized, undistorted point $\mathbf{x}_{n}$ and a coefficient vector $\mathbf{k}=\left(k_{1}, k_{2}, k_{3}, k_{4}\right)^{T}$ as parameters.

The mapping of the distorted, normalized image coordinates $\mathbf{x}_{d}$ to the pixel coordinates $\mathbf{x}$ is computed by

$$
\mathbf{x}^{\prime}=\left(\begin{array}{l}
x^{\prime} \\
y^{\prime} \\
z^{\prime}
\end{array}\right)=\mathbf{K}\left(\begin{array}{c}
\mathbf{x}_{d} \\
1
\end{array}\right) \quad \mathbf{K}=\left(\begin{array}{ccc}
f / s_{x} & 0 & c_{x} \\
0 & f / s_{y} & c_{y} \\
0 & 0 & 1
\end{array}\right)
$$

and

$$
\mathbf{x}=\left(\begin{array}{l}
x \\
y
\end{array}\right)=\frac{1}{z^{\prime}}\left(\begin{array}{l}
x^{\prime} \\
y^{\prime}
\end{array}\right),
$$

where $f$ denotes the focal length of the lens and $s_{x}, s_{y}$ the size of the sensor pixels. The parameters $c_{x}$ and $c_{y}$ specify the image center, i.e., the point where the optical axis hits the sensor layer. In brief, the intrinsic parameters of the camera are given by the camera matrix $\mathbf{K}$ and the distortion parameters $\mathbf{k}=$ $\left(k_{1}, k_{2}, k_{3}, k_{4}\right)^{T}$.

As mentioned in the introduction, each filter wheel position of the multispectral camera is modeled as a single camera system with specific intrinsic parameters. For instance, the parameters for the filter wheel position using an optical bandpass filter with the selected wavelength $\lambda_{\text {sel }}=400 \mathrm{~nm}$ is described by the intrinsic parameters $\mathbf{K}_{\lambda_{\text {sel }}}$ and $\mathbf{k}_{\lambda_{\text {sel }}}$. 


\subsection{Computing the Homography}

In addition to lens distortions, which are mainly characterized by the intrinsic parameters $\mathbf{k}_{\lambda_{\text {sel }}}$, the perspective geometry for each passband is slightly different because of the different optical properties of the bandpass filters: As shown in more detail in 9, a variation of the tilt angle causes an image shift, whereas changes in the thickness or refraction index causes the image to be enlarged or shrunk. Therefore, we have to compute a relation between the image pixel coordinates of the selected passband and the reference passband. The normalized and homogeneous coordinates are derived by

$$
\mathbf{x}_{n, \lambda_{\mathrm{sel}}}=\frac{\mathbf{X}_{\lambda_{\mathrm{sel}}}}{Z_{\lambda_{\mathrm{sel}}}}=\frac{\mathbf{X}_{\lambda_{\mathrm{sel}}}}{\mathbf{e}_{z}^{T} \mathbf{X}_{\lambda_{\mathrm{sel}}}} \quad \text { and } \quad \mathbf{x}_{n, \lambda_{\mathrm{ref}}}=\frac{\mathbf{X}_{\lambda_{\mathrm{ref}}}}{Z_{\lambda_{\mathrm{ref}}}}=\frac{\mathbf{X}_{\lambda_{\mathrm{ref}}}}{\mathbf{e}_{z}^{T} \mathbf{X}_{\lambda_{\mathrm{ref}}}}
$$

respectively, where $\mathbf{X}_{\lambda_{\text {sel }}}$ and $\mathbf{X}_{\lambda_{\text {ref }}}$ are coordinates for the selected and the reference passband. The normalization transforms $\mathbf{X}_{\lambda_{\text {sel }}}$ and $\mathbf{X}_{\lambda_{\text {ref }}}$ to a plane in the position $z_{n, \lambda_{\mathrm{sel}}}=1$ and $z_{n, \lambda_{\mathrm{ref}}}=1$, respectively. In the following, we treat them as homogeneous coordinates, i.e., $\mathbf{x}_{n, \lambda_{\mathrm{sel}}}=\left(x_{n, \lambda_{\mathrm{sel}}}, y_{n, \lambda_{\mathrm{sel}}}, 1\right)^{T}$.

According to our results in [9], where we proved that an affine transformation matrix is well suited to characterize the distortions caused by the bandpass filters solely, we estimate a matrix

$$
\mathbf{H} \mathbf{x}_{n, \lambda_{\text {ref }}}=\mathbf{x}_{n, \lambda_{\text {sel }}} .
$$

The matrix $\mathbf{H}$ transforms coordinates $\mathbf{x}_{n, \lambda_{\text {ref }}}$ from the reference passband to coordinates $\mathbf{x}_{n, \lambda_{\text {sel }}}$ of the selected passband. In practice, we use a set of coordinates from the checkerboard crossing detection during the calibration for reliable estimation of $\mathbf{H}$ and apply a least squares algorithm to solve the overdetermined problem.

\subsection{Performing Rectification}

Finally, the distortions of all passband images have to be compensated and the images have to be adapted geometrically to the reference passband as described in the previous section. Doing this straightforwardly, we would transform the coordinates of a selected passband to the ones of the reference passband. To keep an equidistant sampling in the resulting image this is in practice done the other way round: We start out from the destination coordinates of the final image and compute the coordinates in the selected passband, where the pixel values have to be taken from.

The undistorted, homogeneous pixel coordinates in the target passband are here denoted by $\left(x_{\lambda_{\text {ref }}}, y_{\lambda_{\text {ref }}}, 1\right)^{T}$, the ones of the selected passband are computed by

$$
\left(\begin{array}{c}
u^{\prime} \\
v^{\prime} \\
w^{\prime}
\end{array}\right)=\mathbf{H K}_{\lambda_{\mathrm{ref}}}^{-1}\left(\begin{array}{c}
x_{\lambda_{\mathrm{ref}}} \\
y_{\lambda_{\mathrm{ref}}} \\
1
\end{array}\right),
$$


where $\mathbf{K}_{\lambda_{\text {ref }}}^{-1}$ transforms from pixel coordinates to normalized camera coordinates and $\mathbf{H}$ performs the affine transformation introduced in section 2.2. The normalized coordinates $(u, v)^{T}$ in the selected passband are then computed by

$$
u=\frac{u^{\prime}}{w^{\prime}} \quad v=\frac{v^{\prime}}{w^{\prime}} .
$$

Furthermore, the distorted coordinates are determined using

$$
\left(\begin{array}{c}
\tilde{u} \\
\tilde{v}
\end{array}\right)=f\left(\left(\begin{array}{c}
u \\
v
\end{array}\right), \mathbf{k}_{\lambda_{\text {sel }}}\right),
$$

where $f()$ is the distortion function introduced above and $\mathbf{k}_{\lambda_{\text {sel }}}$ are the distortion coefficients for the selected spectral passband. The camera coordinates in the selected passband are then derived by

$$
\mathbf{x}_{\lambda_{\mathrm{sel}}}=\mathbf{K}_{\lambda_{\mathrm{sel}}}\left(\begin{array}{c}
\tilde{u} \\
\tilde{v} \\
1
\end{array}\right),
$$

where $\mathbf{K}_{\lambda_{\text {sel }}}$ is the camera matrix for the selected passband.

The final warping for a passband image with the wavelength $\lambda_{\text {sel }}$ is done by taking a pixel at the position $\mathbf{x}_{\lambda_{\text {sel }}}$ from the image using bilinear interpolation and storing it at position $\mathbf{x}_{\lambda_{\text {ref }}}$ in the corrected image. This procedure is repeated for all image pixels and passbands.

\section{Results}

A sketch of our multispectral camera is shown in Fig. 1. The camera features a filter wheel with seven optical filters in the range from $400 \mathrm{~nm}$ to $700 \mathrm{~nm}$ in steps of $50 \mathrm{~nm}$ and a bandwidth of $40 \mathrm{~nm}$. The internal grayscale camera is a Sony XCD-SX900 with a resolution of $1280 \times 960$ pixel and a cell size of $4.65 \mu \mathrm{m} \times$ $4.65 \mu \mathrm{m}$. While the internal camera features a C-mount, we use F-mount lenses to be able to place the filter-wheel between sensor and lens. In our experiments, we use a Sigma 10-20mm F4-5.6 lens. Since the sensor is much smaller than a full frame sensor $(36 \mathrm{~mm} \times 24 \mathrm{~mm})$, the focal lengths of the lens has to be multiplied with the crop factor of 5.82 to compute the apparent focal length. This also means that only the center part of the lens is really used for imaging and therefore the distortions are reduced compared to a full frame camera.

For our experiments, we used the calibration chart shown in Fig.2] which comprises a checkerboard pattern with $9 \times 7$ squares and a unit length of $30 \mathrm{~mm}$. We acquired multispectral images for 20 different poses of the chart. Since each multispectral image consists of seven grayscale images representing the passbands, we acquired a total of 140 images. We performed the estimation of intrinsic and extrinsic parameters with the well-known Bouguet toolbox [16] for each passband separately, i.e., we obtain seven parameter datasets. The calibration is then done using the equations in section 2, In this paper, the multispectral images, which 


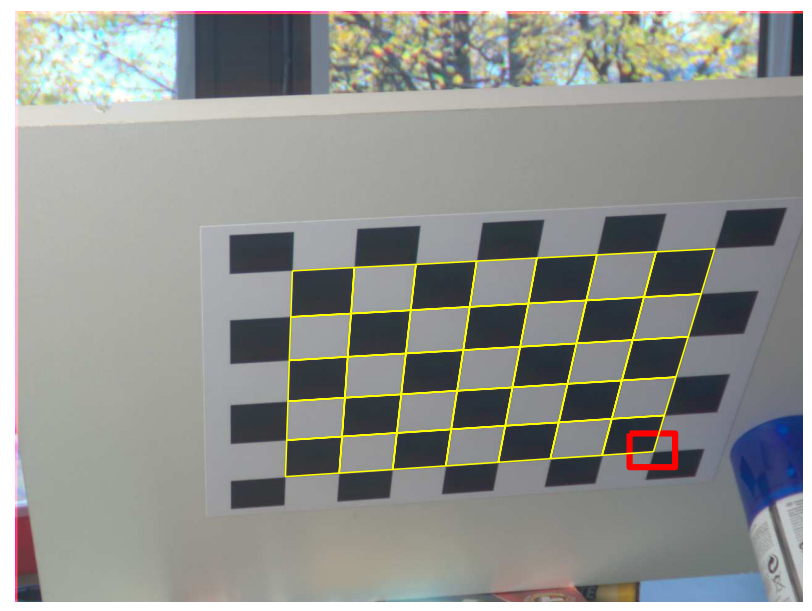

Fig. 2. Exemplary calibration image; distortions have been compensated with the proposed algorithm. The detected checkerboard pattern is marked with a grid. The small rectangle marks the crop area shown enlarged in Fig. 3.

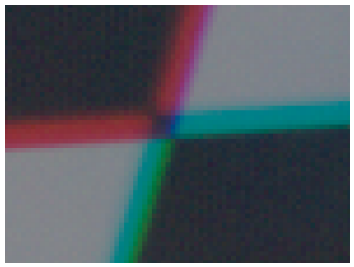

(a) Without geometric calibration color fringes are not compensated.

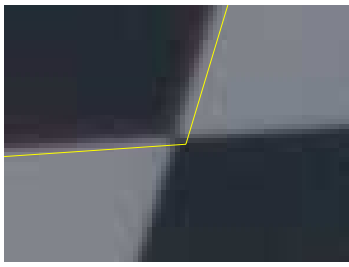

(b) Calibration shown in 9]: color fringes are removed but lens distortions remain.

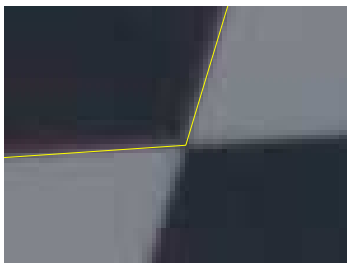

(c) Proposed calibration scheme: both color fringes and lens distortions are removed.

Fig. 3. Crops of the area shown in Fig. 2 for different calibration algorithms

consist of multiple grayscale images, are transformed to the sRGB color space for visualization. Details of this procedure are, e.g., given in [17.

When the geometric calibration is omitted, the final RGB image shows large color fringes as shown in Fig. 3a, Using our previous calibration algorithm in [9], the color fringes vanish (see Fig. $3 \mathrm{~b}$ ), but lens distortions still remain: The undistorted checkerboard squares are indicated by thin lines in the magnified image; the corner of the lines is not aligned with the underlying image, and thus shows the distortion of the image. Small distortions might be acceptable for several imaging tasks, where geometric accuracy is rather unimportant. However, e.g., industrial machine vision tasks often require a distortion-free image, which can be computed by our algorithm. The results are shown in Fig. 3c, where the edge of the overlayed lines is perfectly aligned with the checkerboard crossing of the underlying image. 
Table 1. Reprojection errors in pixels for all spectral passbands. Each entry shows the mean of Euclidean length and maximum pixel error, separated with a slash. For a detailed explanation see text.

\begin{tabular}{|l|l|l|l|l|l|l|l||l|}
\hline & $400 \mathrm{~nm}$ & $450 \mathrm{~nm}$ & $500 \mathrm{~nm}$ & $550 \mathrm{~nm}$ & $600 \mathrm{~nm}$ & $650 \mathrm{~nm}$ & $700 \mathrm{~nm}$ & all \\
\hline no calib. & $2.0 / 4.9$ & $1.2 / 2.6$ & $0.6 / 2.2$ & $0.0 / 0.0$ & $5.0 / 5.4$ & $2.2 / 3.3$ & $3.8 / 7.0$ & $2.11 / 6.97$ \\
\hline intra-band & $0.1 / 0.6$ & $0.1 / 0.6$ & $0.1 / 0.6$ & $0.1 / 0.6$ & $0.1 / 0.6$ & $0.1 / 0.5$ & $0.1 / 0.6$ & $0.10 / 0.61$ \\
\hline inter-band & $0.1 / 0.7$ & $0.1 / 0.6$ & $0.2 / 0.9$ & $0.1 / 0.6$ & $0.2 / 0.8$ & $0.1 / 0.7$ & $0.2 / 0.7$ & $0.14 / 0.91$ \\
\hline
\end{tabular}

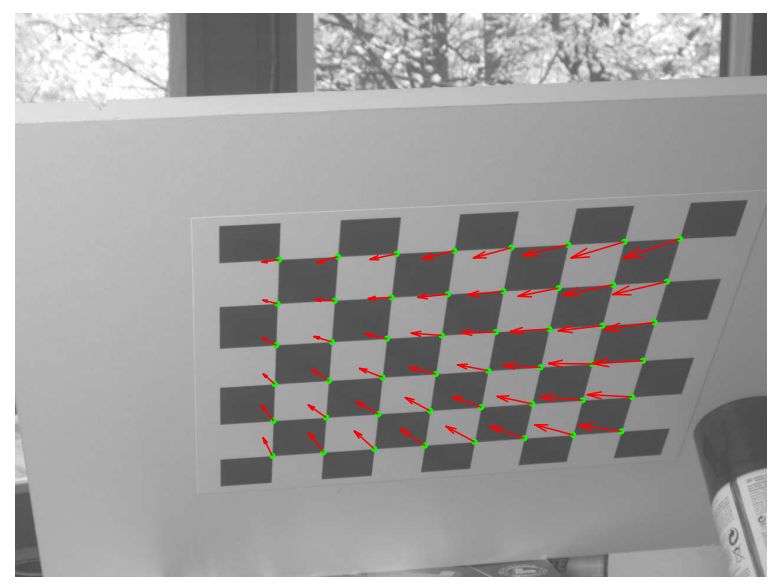

Fig. 4. Distortions caused by the bandpass filters; calibration pattern pose 11 for passband $550 \mathrm{~nm}$ (reference passband); scaled arrows indicate distortions between this passband and the $500 \mathrm{~nm}$ passband

Table 1 shows reprojection errors for all spectral passbands from $400 \mathrm{~nm}$ to $700 \mathrm{~nm}$ and a summary in the last column "all". The second row lists the deviations when no calibration is performed at all. For instance, the fourth column denotes the mean and maximum distances (separated with a slash) of checkerboard crossings between the $500 \mathrm{~nm}$ and the $550 \mathrm{~nm}$ passband: This means, in the worst case, the checkerboard crossing in the $500 \mathrm{~nm}$ passband is located 2.2 pixel away from the corresponding crossing in the $550 \mathrm{~nm}$ passband. In other words, the color fringe in the combined image has a width of 2.2 pixel at this location, which is not acceptable. The distortions are also shown in Fig. 4.

The third row "intra-band" indicates the reprojection errors between the projection of 3D points to pixel coordinates via Eqs. (11)-(5) and their corresponding measured coordinates. We call these errors "intra-band" because only differences in the same passband are taken into account; the differences show how well the passband images can be calibrated themselves, without considering the geometrical connection between them. Since the further transformation via a homography introduces additional errors, the errors given in the third row mark a theoretical limit for the complete calibration (fourth row). 
In contrast to the "intra-band" errors, the "inter-band" errors denoted in the fourth row include errors caused by the homography between different spectral passbands. More precisely, we computed the difference between a projection of $3 \mathrm{D}$ points in the reference passband to pixel coordinates in the selected passband and compared them to measured coordinates in the selected passband. These numbers show how well the overall model is suited to model the multispectral camera, i.e., the deviation which remains after calibration. The mean overall error of 0.14 pixels for all passbands lies in the subpixel range. Therefore, our algorithm is well suited to model the distortions of the multispectral camera. The intra and inter band errors (third and fourth row) for the $550 \mathrm{~nm}$ reference passband are identical because no homography is required here and thus no additional errors are introduced.

Compared to our registration algorithm presented in [9], the algorithm shown in this paper is able to compensate for lens distortions as well. As a side-effect, we also gain information about the focal length and the image center, since both properties are computed implicitly by the camera calibration. However, the advantage of [9] is that almost every image can be used for calibration - there is no need to perform an explicit calibration with a dedicated test chart, which might be time consuming and not possible in all situations. Also, the algorithms for camera calibration mentioned in this paper are more complex, although most of them are provided in toolboxes. Finally, for our specific configuration, the lens distortions are very small. This is due to a high-quality lens and because we use a smaller sensor (C-mount size) than the lens is designed for (F-mount size); therefore, only the center part of the lens is used.

\section{Conclusions}

We have shown that both color fringes caused by the different optical properties of the color filters in our multispectral camera as well as geometric distortions caused by the lens can be corrected with our algorithm. The mean absolute calibration error for our multispectral camera is 0.14 pixel, and the maximum error is 0.91 pixel for all passbands. Without calibration, mean and maximum errors are 6.97 and 2.11, respectively. Our framework is based on standard tools for camera calibration; with these tools, our algorithm can be implemented easily.

\section{Acknowledgments}

The authors are grateful to Professor Bernhard Hill and Dr. Stephan Helling, RWTH Aachen University, for making the wide angle lens available.

\section{References}

1. Yamaguchi, M., Haneishi, H., Ohyama, N.: Beyond Red-Green-Blue (RGB): Spectrum-based color imaging technology. Journal of Imaging Science and Technology 52(1), 010201-1-010201-15 (2008) 
2. Luther, R.: Aus dem Gebiet der Farbreizmetrik. Zeitschrift für technische Physik 8, 540-558 (1927)

3. Hill, B., Vorhagen, F.W.: Multispectral image pick-up system, U.S.Pat. 5,319,472, German Patent P 4119489.6 (1991)

4. Tominaga, S.: Spectral imaging by a multi-channel camera. Journal of Electronic Imaging 8(4), 332-341 (1999)

5. Burns, P.D., Berns, R.S.: Analysis multispectral image capture. In: IS\&T Color Imaging Conference, Springfield, VA, USA, vol. 4, pp. 19-22 (1996)

6. Mansouri, A., Marzani, F.S., Hardeberg, J.Y., Gouton, P.: Optical calibration of a multispectral imaging system based on interference filters. SPIE Optical Engineering 44(2), 027004.1-027004.12 (2005)

7. Haneishi, H., Iwanami, T., Honma, T., Tsumura, N., Miyake, Y.: Goniospectral imaging of three-dimensional objects. Journal of Imaging Science and Technology 45(5), 451-456 (2001)

8. Brauers, J., Aach, T.: Longitudinal aberrations caused by optical filters and their compensation in multispectral imaging. In: IEEE International Conference on Image Processing (ICIP 2008), San Diego, CA, USA, pp. 525-528. IEEE, Los Alamitos (2008)

9. Brauers, J., Schulte, N., Aach, T.: Multispectral filter-wheel cameras: Geometric distortion model and compensation algorithms. IEEE Transactions on Image Processing 17(12), 2368-2380 (2008)

10. Cappellini, V., Del Mastio, A., De Rosa, A., Piva, A., Pelagotti, A., El Yamani, H.: An automatic registration algorithm for cultural heritage images. In: IEEE International Conference on Image Processing, Genova, Italy, September 2005, vol. 2, pp. II-566-9 (2005)

11. Kern, J.: Reliable band-to-band registration of multispectral thermal imager data using multivariate mutual information and cyclic consistency. In: Proceedings of SPIE, November 2004, vol. 5558, pp. 57-68 (2004)

12. Helling, S., Seidel, E., Biehlig, W.: Algorithms for spectral color stimulus reconstruction with a seven-channel multispectral camera. In: IS\&Ts Proc. 2nd European Conference on Color in Graphics, Imaging and Vision CGIV 2004, Aachen, Germany, April 2004, vol. 2, pp. 254-258 (2004)

13. Hartley, R.I., Zisserman, A.: Multiple View Geometry in Computer Vision, 2nd edn. Cambridge University Press, Cambridge (2004)

14. Gao, C., Ahuja, N.: Single camera stereo using planar parallel plate. In: Ahuja, N. (ed.) Proceedings of the 17th International Conference on Pattern Recognition, vol. 4, pp. 108-111 (2004)

15. Gao, C., Ahuja, N.: A refractive camera for acquiring stereo and super-resolution images. In: Ahuja, N. (ed.) IEEE Computer Society Conference on Computer Vision and Pattern Recognition, New York, USA, vol. 2, pp. 2316-2323 (2006)

16. Bouguet, J.Y.: Camera Calibration Toolbox for Matlab

17. Brauers, J., Schulte, N., Bell, A.A., Aach, T.: Multispectral high dynamic range imaging. In: IS\&T/SPIE Electronic Imaging, San Jose, California, USA, January 2008, vol. 6807 (2008) 